\title{
Noninvasive Brain Stimulation for Motor Recovery after Stroke: Mechanisms and Future Views
}

\author{
Naoyuki Takeuchi and Shin-Ichi Izumi \\ Department of Physical Medicine and Rehabilitation, Tohoku University School of Medicine, 2-1 Seiryo-cho, Aoba-ku, \\ Sendai 980-8575, Japan \\ Correspondence should be addressed to Naoyuki Takeuchi, naoyuki@med.hokudai.ac.jp
}

Received 20 July 2012; Accepted 29 August 2012

Academic Editor: Claudia Altamura

Copyright ( $\odot 2012$ N. Takeuchi and S.-I. Izumi. This is an open access article distributed under the Creative Commons Attribution License, which permits unrestricted use, distribution, and reproduction in any medium, provided the original work is properly cited.

\begin{abstract}
Repetitive transcranial magnetic stimulation and transcranial direct current stimulation are noninvasive brain stimulation (NIBS) techniques that can alter excitability of the human cortex. Considering the interhemispheric competition occurring after stroke, improvement in motor deficits can be achieved by increasing the excitability of the affected hemisphere or decreasing the excitability of the unaffected hemisphere. Many reports have shown that NIBS application improves motor function in stroke patients by using their physiological peculiarity. For continuous motor improvement, it is important to impart additional motor training while NIBS modulates the neural network between both hemispheres and remodels the disturbed network in the affected hemisphere. NIBS can be an adjuvant therapy for developed neurorehabilitation strategies for stroke patients. Moreover, recent studies have reported that bilateral NIBS can more effectively facilitate neural plasticity and induce motor recovery after stroke. However, the best NIBS pattern has not been established, and clinicians should select the type of NIBS by considering the NIBS mechanism. Here, we review the underlying mechanisms and future views of NIBS therapy and propose rehabilitation approaches for appropriate cortical reorganization.
\end{abstract}

\section{Introduction}

Stroke is the major cause of disability worldwide $[1,2]$. A number of neurological functions are impaired by stroke; the most common impairment is motor disability contralateral to the stroke lesion side [3]. Despite rehabilitation, the motor function outcome after stroke is often incomplete, and dexterity deficits are a considerable handicap to stroke survivors [1]. Therefore, various strategies are developing that aim to enhance motor recovery [4-6]. Motor recovery after stroke is related to neural plasticity, which refers to the ability of the brain to develop new neuronal interconnections, acquire new functions, and compensate for impairment $[7,8]$. The mechanisms of neural plasticity in motor function recovery after stroke are well documented for both animal and human models. Reorganization induced by neural plasticity includes modulation of neural activation within the remaining motor area network to maximize neural resources for motor recovery [7, 9-11].
Repetitive transcranial magnetic stimulation (rTMS) and transcranial direct current stimulation (tDCS) are noninvasive brain stimulation (NIBS) techniques that can alter human cortex excitability [12]. Transcranial magnetic stimulation (TMS) is a non-invasive method of stimulating the cortex through the scalp and skull. TMS involves using a wire coil placed over the scalp to generate a short-lasting, local magnetic field. When the pulsed magnetic field enters the brain, it creates an electrical current that flows through neurons, inducing neuronal depolarization. rTMS is defined as repetition of TMS; high-frequency rTMS increases cortical excitability, whereas low-frequency rTMS suppresses cortical excitability [12]. Theta burst stimulation (TBS) has also been reported as an effective rTMS method. It uses repeating bursts of very low intensity combined-frequency rTMS [13]. Each burst consists of 3 stimuli (delivered at $50 \mathrm{~Hz}$ ) repeating at $5 \mathrm{~Hz}$. TBS can be used in 2 ways: a continuous train is used to suppress cortical excitability and an intermittent pattern is used to enhance cortical excitability. tDCS is another 
commonly used NIBS technique. Although compared to rTMS, tDCS has been studied for much longer in animals, only recently have the tDCS mechanisms of action been investigated in human studies [14]. tDCS does not cause neuronal depolarization. Rather, it modulates the neuronal membrane potential through polarizing currents by weak constant direct current, thereby influencing the levels of excitability and modulating the spontaneous firing rate of neurons. There are 2 types of tDCS: anodal tDCS increases the excitability of the stimulated cortex, whereas cathodal tDCS decreases the excitability of the stimulated cortex [15].

Many reports have shown that NIBS techniques help improve the efficacy of rehabilitative strategies employed after stroke by using physiological peculiarity that can alter the cortical excitability. The idea is that modulation of cortical excitability may induce neural plasticity and/or interfere with maladaptive neural activation, which subsequently weakens motor function and limits motor recovery [16]. However, the mechanisms underlying motor recovery after NIBS therapy remain to be elucidated, and effective NIBS methods remain insufficiently proven or widespread. This review focuses on 4 important factors regarding NIBS for stroke patients with hemiparesis: (1) the mechanism of NIBS therapy for motor recovery, (2) inhibitory and excitatory NIBS, (3) the influence of clinical factors on effects of NIBS, and (4) combination of NIBS with other therapies and bilateral NIBS. The purpose of this paper is to provide a comprehensive overview of NIBS for motor stroke, to understand its mechanisms, and suggest approaches for appropriate cortical reorganization.

\section{Mechanism of NIBS Therapy for Motor Recovery}

NIBS therapy for motor stroke aims to augment neural plasticity and improve motor function based on the interhemispheric competition model. The interhemispheric competition model proposes that motor deficits in stroke patients are due to reduced output from the affected hemisphere and excessive interhemispheric inhibition from the unaffected hemisphere to the affected hemisphere [17, 18]. Therefore, using NIBS, improvement in motor deficits can be achieved by increasing the excitability of the affected hemisphere or decreasing the excitability of the unaffected hemisphere $[19,20]$. No relevant adverse effects of NIBS, such as epileptic seizure induction, have been reported to occur in stroke patients when current safety guidelines regarding the intensity, frequency, and time of stimulation are adhered to $[21,22]$. So far, no study has reported different effects of rTMS and tDCS on motor improvement in stroke patients. Therefore, in this section, we discuss the mechanism of the NIBS therapy for motor recovery after stroke, without distinguishing between rTMS and tDCS.

2.1. NIBS Modulates Cortical Neural Plasticity. Several studies have reported the neurophysiological changes in stroke patients after NIBS using TMS techniques [18, 23-26]. Inhibitory NIBS increases excitability in the ipsilesional motor cortex by reducing the excessive interhemispheric inhibition from the contralesional motor cortex [18, 25]. Excitatory NIBS over the affected hemisphere directly increases the excitability of the ipsilesional motor cortex $[23,24,26]$. Excitability enhancement in the motor cortex appears to be required for motor learning [27, 28]. Therefore, NIBS can facilitate motor learning and induce motor recovery by directly or indirectly increasing the excitability in the ipsilesional motor cortex. In fact, compared to motor training or NIBS alone, pairing motor training with NIBS results in prolonged performance improvements and functional neural plasticity in the ipsilesional motor cortex [18-20, 25]. Studies using functional neuroimaging have found that cerebral blood flow of the ipsilesional motor cortex is increased after inhibitory NIBS over the unaffected hemisphere and excitatory NIBS over the affected hemisphere $[29,30]$. These NIBS-induced metabolic changes may also promote neural plasticity and motor recovery after stroke [29]. Moreover, excitatory NIBS over the affected hemisphere can induce long-term potentiation-like changes in the affected hemisphere and promote motor recovery after stroke [26]. Therefore, NIBS may resolve impairment of experience-dependent plasticity in the affected hemisphere after stroke [16, 31, 32]. Furthermore, cumulative NIBS has been shown to be important for continuous motor improvement $[33,34]$. This result indicates that neural plasticity is consolidated by cumulative NIBS intervention. These findings suggest that artificially modulating the cortical excitability by NIBS may induce a more suitable environment for neural plasticity. NIBS can be an important technique in the rehabilitation of stroke patients; providing motor training along with NIBS and conducting NIBS cumulatively will help sustain the effect of NIBS and improve motor function.

2.2. Modulating the Neural Network in Stroke Patients. In addition to excitability modulation in the ipsilesional motor cortex, NIBS may modulate the neural network in both hemispheres to induce motor recovery. Studies using functional magnetic resonance imaging and electroencephalogram may help to elucidate the effects of NIBS therapy for stroke on the motor cortical network. A neuroimaging study revealed that inhibitory NIBS over the unaffected hemisphere reduced the pathological hyperactivity in the primary and nonprimary motor cortices in the unaffected hemisphere [35]. Excitatory NIBS over the affected hemisphere has been shown to reduce neural activity in the contralesional motor cortex, in addition to facilitation of the ipsilesional motor cortex [30]. Moreover, inhibitory NIBS over the unaffected hemisphere reduced the connectivity of both hemispheres and enhanced coupling between the primary and nonprimary motor cortices in the affected hemisphere $[36,37]$. Enhanced excitability in the unaffected hemisphere inhibits the affected hemisphere via excessive interhemispheric inhibition and weakens motor function of the paretic side [17]. Moreover, it has been reported that the nonprimary motor cortices may contribute to motor recovery after stroke $[38,39]$. Although the change in neural coupling after excitatory NIBS remains unclear, normalized 


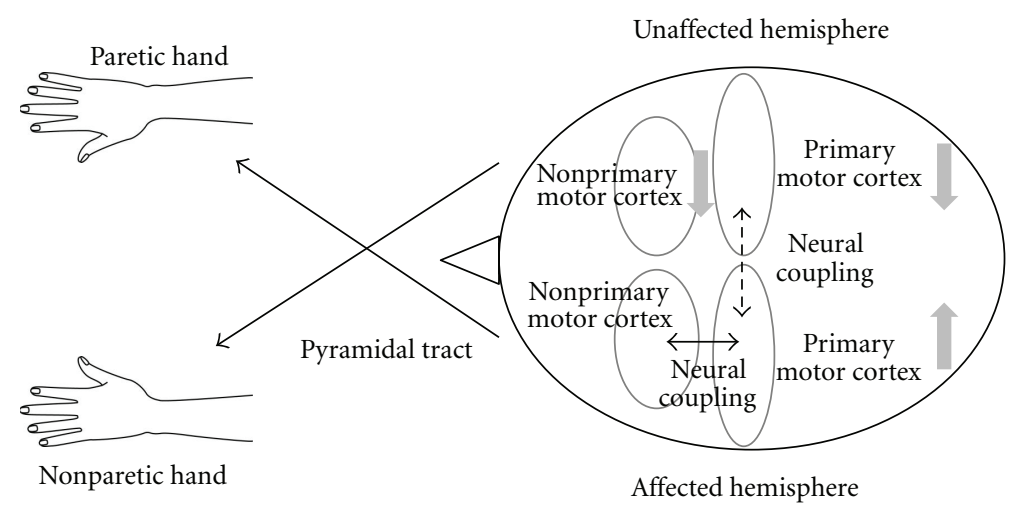

FIGURE 1: Mechanism of noninvasive brain stimulation therapy in stroke patients. Noninvasive brain stimulation (NIBS) increases ipsilesional primary motor cortex excitability and improves motor function of the paretic side by ameliorating impaired experiencedependent plasticity in stroke patients. NIBS reduces hyperactivity in the primary and nonprimary motor cortices in the unaffected hemisphere and neural coupling of both hemispheres. Moreover, NIBS enhances neural coupling between the primary and nonprimary motor cortices in the affected hemisphere. In addition to facilitation of the ipsilesional primary motor cortex, excitability modulation in both hemispheres, and reconstructed neural coupling between the primary and nonprimary motor cortices in the affected hemisphere after NIBS contribute to motor recovery in stroke patients.

excitability of both hemispheres and reconstruction of effective connectivity between the primary and nonprimary motor cortices in the affected hemisphere after NIBS may contribute to motor recovery in stroke patients (Figure 1).

\section{Inhibitory and Excitatory NIBS}

An important concern for clinicians and stroke patients is what kind of stimulation parameter is most effective for motor recovery. A recent meta-analysis study of rTMS on upper limb motor function in stroke patients reported that low-frequency rTMS over the unaffected hemisphere might be more beneficial than high-frequency rTMS over the affected hemisphere [40]. Although more researchers have begun to evaluate the effectiveness of different NIBS stimulation protocols for motor recovery after stroke, further well-designed studies in larger populations are required to identify the most effective types of NIBS from various protocols, including tDCS for stroke treatment. Therefore, at present, clinicians have to select the NIBS type by considering the advantages and disadvantages. In this section, we broadly divide NIBS into 2 types inhibitory and excitatory considering the interhemispheric competitive model. We describe the peculiarity of inhibitory and excitatory NIBS for motor stroke and discuss the optimal stimulation intensities for inhibitory and excitatory NIBS.

3.1. Inhibitory NIBS over the Unaffected Hemisphere. An advantage of NIBS over the unaffected hemisphere is that its response is more uniform than of stimulation over the affected hemisphere, because the unaffected site is less likely to be affected by neuronal loss or tissue damage [41]. Moreover, inhibitory NIBS over the unaffected hemisphere is expected to be safer with respect to any potential seizure risk (particularly in case of rTMS) or tissue damage [22]. However, inhibitory NIBS has a risk of deteriorating some motor functions by reducing excitability of the stimulated cortex. One possible risk is the deterioration of nonparetic motor function that may occur by downregulation of the contralesional motor cortex. However, several studies reported that inhibitory NIBS over the unaffected hemisphere did not change the motor function of the nonparetic side $[35,42]$. Second, inhibitory NIBS therapy may deteriorate the motor function of the paretic side itself by disrupting the ipsilateral motor projections that are enhanced after stroke [43-45]. However, the ipsilateral motor projections are insufficient to support motor recovery, especially for distal muscles because the distal muscles are primarily innervated by contralateral corticospinal projections [46], whereas ipsilateral motor projections to the distal muscles are scarce [47]. Therefore, it is unlikely that inhibitory NIBS over the unaffected hemisphere deteriorates the motor function of the paretic side, particularly the distal side. However, activation of ipsilateral motor projections has been suggested to be beneficial for trunk muscle movement and children [48-50]. Therefore, future studies on inhibitory NIBS are needed to clarify the possible risk of deterioration in trunk muscle movement or motor function in children. Finally, it was noted that inhibitory NIBS reduces the interhemispheric inhibition that controls bimanual movement $[51,52]$. In fact, a recent study reported that inhibitory rTMS over the unaffected hemisphere deteriorated the performance in the antiphase bimanual tapping task in stroke patients [42]. Therefore, inhibitory NIBS may deteriorate bimanual movement by reducing the interhemispheric inhibition that controls bimanual movement (Figure 2(a)).

3.2. Excitatory NIBS over the Affected Hemisphere. Although excitatory NIBS over the affected hemisphere has the advantage that it does not inhibit the stimulation site, its effect is dependent on the anatomical changes in the affected hemisphere. Damaged brain tissue evolves into 


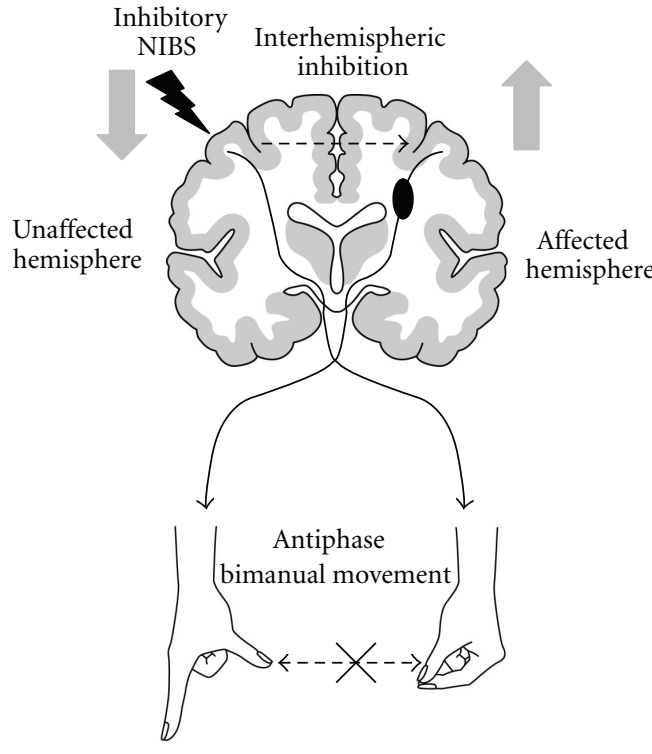

Paretic hand

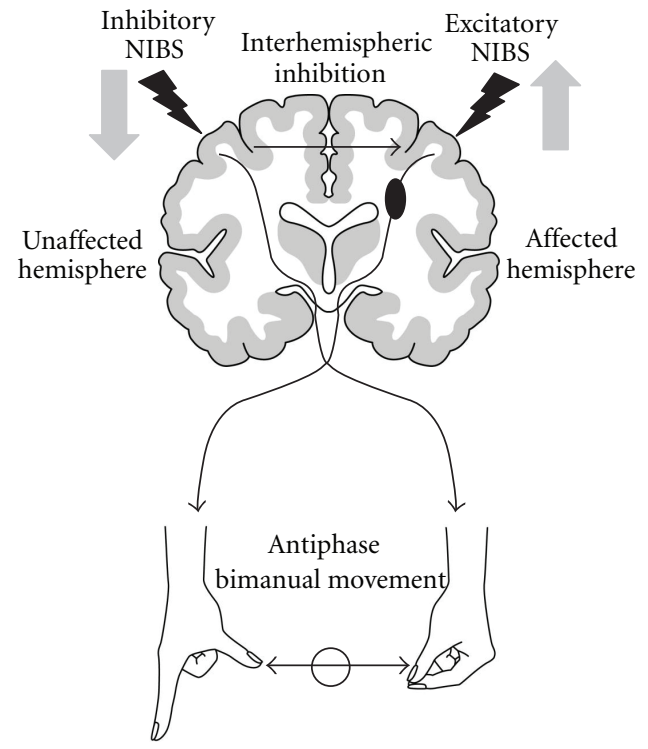

Paretic hand

(a)

(b)

FIGURE 2: Mechanism and prevention of bimanual movement deterioration by NIBS in stroke patients. (a) Inhibitory NIBS over the unaffected hemisphere. Inhibitory NIBS decreases excitability of the contralesional motor cortex and increases excitability of the ipsilesional motor cortex by reducing interhemispheric inhibition from the unaffected to the affected hemisphere. Antiphase bimanual movement deteriorates due to the reduction in interhemispheric inhibition, which controls bimanual movement. (b) Bilateral NIBS. Excitatory NIBS, along with inhibitory NIBS, decreases excitability of the contralesional motor cortex and increases excitability of the ipsilesional motor cortex. However, bilateral NIBS limits the reduction in interhemispheric inhibition induced by inhibitory NIBS and prevents antiphase bimanual movement deterioration. Modified from Takeuchi et al. [42].

scar tissue and is replaced by cerebral spinal fluid (CSF) spaces (particularly in cortical damage); scar formation and large CSF spaces inhibit the effect of NIBS because the conductance of the CSF is 4 to 10 times higher than that of normal brain tissue $[53,54]$. Therefore, careful modeling using a stereotactic system with integrated anatomical data is required to predict the effect of excitatory NIBS over the affected hemisphere [53-55]. Moreover, it was noted that excitatory NIBS over the affected hemisphere slightly deteriorated the dexterity of the paretic hand in some cortical stroke patients [30]. Although in excitatory NIBS over the unaffected hemisphere, the stimulation site is not in the affected hemisphere, it may be beneficial for some motor functions due to activation of ipsilateral motor projections. A recent study reported that excitatory NIBS over the unaffected hemisphere improved swallowing, which involves ipsilateral motor projections [41]. Therefore, excitatory NIBS over the unaffected hemisphere may improve trunkmuscle movement or motor function in children, which is strongly innervated by ipsilateral motor projections.

\subsection{Optimal Stimulation Intensity for Inhibitory and Excita-} tory NIBS. The frequency, intensity, and number of pulses are important factors for rTMS effects, and the amplitude and stimulation duration are important factors for tDCS effects. However, the most effective parameters for motor recovery after stroke remain to be elucidated. In healthy controls, several studies have reported that both inhibitory and excitatory NIBS with strong intensity and long duration (numerous numbers) more effectively induce neurophysiological changes $[15,56-59]$. Some previous studies reported that excitatory NIBS over the affected hemisphere with insufficient stimulation power might not lead to motor recovery in stroke patients $[20,42]$. Therefore, it is likely that excitatory NIBS with strong intensity, numerous numbers, and long duration are most effective for motor recovery. However, for inhibitory NIBS over the unaffected hemisphere, strong stimulation intensity may not necessarily be the best parameter for motor recovery. In rTMS studies of healthy controls, the $90 \%$ rest motor threshold (rMT), but not $115 \%$ rMT or $150 \%$ rMT, showed significant motor improvement ipsilateral to the stimulated hemisphere [6062]. A subthreshold stimulation to the rMT may act via local inhibition of the stimulation site, whereas suprathreshold stimulation ( 115 or $150 \%$ rMT) may inhibit the stimulation site as well as the opposite homogenous motor cortex via interhemispheric inhibition. Activation of interhemispheric inhibition by suprathreshold stimulation may cancel out the facilitation effect on the contralateral motor cortex due to reduced excitability of the stimulated motor cortex, resulting in unchanged motor function. Therefore, for inhibitory NIBS over the unaffected hemisphere, excessive stimulation intensity may be ineffective for motor recovery in stroke patients. 


\section{Influence of Clinical Factors on the Effect of NIBS}

A few studies have clarified which stroke patients are more responsive to NIBS therapy $[30,63]$. Therefore, we must estimate the effect of NIBS therapy for stroke patients from studies that revealed the mechanism of NIBS therapy. In this section, we will discuss whether age, stroke duration, lesion site, or severity of motor function loss affects NIBS therapy.

4.1. Age. Age is an important factor for effect of NIBS therapy. It has been reported that NIBS could improve motor recovery in adults as well as children [64]. However, the corpus callosum is not formed until 6-8 years of age [65]. Therefore, it is unlikely that inhibitory NIBS over the unaffected hemisphere will improve the motor function of the paretic side in children younger than 6-8 years, because inhibitory NIBS improves motor recovery by reducing the excessive interhemispheric inhibition to the affected hemisphere [18]. Moreover, the ipsilateral motor projections activated after stroke may assist motor recovery in children more than adults $[49,50]$. Therefore, it should be noted that inhibitory NIBS over the unaffected hemisphere might be less effective or even harmful in young children. In addition, the long-term effect of excitatory NIBS in young children, particularly epileptic induction, remains to be determined [22]. Therefore, clinicians should be careful with NIBS therapy for young children with stroke. In addition, NIBS therapy might be less effective for elderly stroke patients [63, 66 ] because elderly patients have lower motor learning ability and neural plasticity induced by NIBS $[66,67]$. However, a previous study has reported no correlation between motor improvement after NIBS and age [30], so future studies need to closely examine the effect of NIBS therapy on patients of various ages.

4.2. Stroke Duration. It is important to determine whether NIBS therapy is more effective in the acute or chronic stage after stroke. If NIBS therapy is more effective in acute stages, early modulation of cortical excitability may facilitate motor recovery and prevent development of maladaptive neural plasticity by rebalancing interhemispheric communication and normalizing neural activity within the motor areas of both hemispheres. Although it has been reported that both inhibitory and excitatory NIBS facilitates motor recovery in stroke patients at the acute stage $[26,68-70]$, a recent study showed that inhibitory NIBS did not facilitate motor recovery in patients in acute stages of stroke [71]. Moreover, it is speculated that perilesional activity increases oxygen and glucose demands in the stroke penumbra and thus induces extension of the lesion $[72,73]$. Therefore, any procedure increasing cortical excitability should be performed with caution during the acute stages after stroke [26]. Further investigations are required to determine whether NIBS in the acute stroke stage can promote the final motor function.

4.3. Lesion Site and Neural Network. The lesion site may also influence the effect of NIBS therapy. Several studies on excitatory NIBS over the affected hemisphere reported no difference in motor improvement between patients with subcortical and cortical stroke lesions [24, 33, 74, 75]. However, a study showed that excitatory NIBS over the affected hemisphere improved dexterity of the paretic hand in subcortical stroke patients but not in some cortical stroke patients [30]. As well as difference of behavioral change between subcortical and cortical stroke, excitatory rTMS over the affected hemisphere reduced neural activity of the contralesional motor cortex in patients with subcortical stroke but caused widespread bilateral recruitment of primary and nonprimary motor areas in patients with cortical stroke [30]. It may be too simple to distinguish stroke patients by cortical and subcortical lesion. However, the common target of both inhibitory and excitatory NIBS is ipsilesional motor cortex activation. Therefore, NIBS therapy might be less effective for cortical stroke patients, particularly in the ipsilesional motor cortex. The evaluation of corticospinal tract integrity also may be useful to predict the benefits of NIBS.

In addition to stroke lesion site, the pattern of neural network activation in both hemispheres may influence the effect of NIBS therapy for motor stroke patients. It was reported that good responders for inhibitory NIBS over the unaffected hemisphere have hyperactive contralesional dorsal premotor cortex and contralesional parietal operculum before stimulation [35]. On the other hand, a previous study reported that ipsilesional motor cortex excitability in good responders for excitatory NIBS over the affected hemisphere is easily facilitated by moving the paretic hand before stimulation [30]. Therefore, identifying patients based on individual patterns of cortical activation may help select patients most suited for NIBS therapy after stroke.

4.4. Severity of Motor Function Loss. It is unknown whether the effect of NIBS therapy on stroke depends on the severity of motor function loss. Most NIBS studies in stroke focused on patients with mild or moderate motor deficits that had recovered enough to perform required motor function tests. Favorable motor recovery is achieved if patients can perform motor training by themselves to sustain the effect of NIBS, because it is important to perform motor training after NIBS for continuous motor improvement [25]. However, a previous case study reported that stroke patient with severe hemiparesis could move the hand after NIBS therapy [76]. In addition to motor improvement, NIBS therapy may ameliorate the paretic side spasticity $[77,78]$. Therefore, if not contraindicated for other reasons, NIBS therapy may be worth trying in stroke patients with severe hemiparesis.

Most studies with NIBS therapy have focused on the upper limbs $[18,20,23-26]$ and the effect of NIBS on the lower limbs of stroke patients has been rarely tested. Recent studies reported that excitatory tDCS over the affected hemisphere improves knee extensor strength and motor training effect in ankle tracking tasks in stroke patients $[79,80]$. To our knowledge, there are no studies showing that inhibitory NIBS can improve paretic lower limb motor function in stroke patients. However, in a previous study, inhibitory rTMS over the unaffected motor cortex increased corticospinal excitability of the paretic lower 
limb [81]. Therefore, inhibitory NIBS over the unaffected hemisphere may also improve the motor function of the lower limb. However, compared to the hand motor area, the lower limb area has fewer inhibitory circuits $[82,83]$. Thus, the effect of inhibitory NIBS for lower limbs might be different from those for upper limbs. It is noted that inhibitory NIBS for the lower limb may inhibit the affected hemisphere itself, because the lower limb motor cortices on either side of the mid-sagittal fissure are closer than the upper limb cortices. Future studies should be conducted to elucidate whether focal inhibitory NIBS over the unaffected hemisphere improves motor function in the lower limb.

\section{Combination of NIBS with other Therapies and Bilateral NIBS}

Various neurorehabilitation strategies are emerging to enhance beneficial plasticity and improve motor recovery [4-6]. As mentioned above, NIBS can modulate cortical excitability, so NIBS might be an adjuvant therapy for developed neurorehabilitation strategies. Moreover, recent studies have reported modified NIBS methods to promote motor recovery [20, 42, 84-86]. In this section, we will discuss the combination of NIBS with neurorehabilitation techniques and recent bilateral NIBS methods.

5.1. Combination of NIBS with Other Therapies. Several studies showed that the combination of NIBS with specific neurorehabilitation techniques improved motor recovery. Preliminary studies have reported the combination therapy of NIBS with robotic motor training after stroke [87, 88]. A controlled study reported that the combination of NIBS with neuromuscular stimulation showed more improvement in motor recovery than NIBS or neuromuscular stimulation alone [89]. In addition, it has been reported that combining NIBS with constraint-induced motor therapy (CIMT) had an additional effect on motor recovery [90]. In contrast, some controlled studies reported that the combining NIBS with CIMT or neuromuscular stimulation had no additional effect on motor recovery [91, 92]. The number of these combination studies is still small, and they are preliminary and controversial. Although it is likely that NIBS promotes the effects of developed neurorehabilitation strategies for stroke patients, future studies are required to determine the appropriate combination of methods for motor recovery. As another potential strategy, the combination of NIBS with pharmacotherapy may facilitate motor recovery in stroke patients. Several studies have shown that pharmacologic agents can modulate neural excitability in response to NIBS techniques $[66,93]$. In healthy controls, the main drugs that increase the effects of NIBS are N-methyl-d-aspartate receptor agonists [94], levodopa [95], dopamine agonists [96, 97], nicotine [98], amphetamine [99], and selective serotonin reuptake inhibitors [100]. However, it is unclear how these drugs influence neural excitability in response to NIBS in stoke patients. Further, we must consider the possibility that the response to pharmacological approaches is different between stroke patients and healthy controls [66].
5.2. Bilateral NIBS. As mentioned above, the common aim of inhibitory and excitatory NIBS is activation of the ipsilesional motor cortex and rebalancing both hemispheres. Therefore, considering the interhemispheric competitive model, it may be more suitable for motor recovery to stimulate both hemispheres using inhibitory and excitatory NIBS. Several recent studies have reported that compared to unilateral NIBS, simultaneous bilateral NIBS using rTMS and tDCS improves motor recovery more effectively in stroke patients [42, 85, 86]. Moreover, bilateral NIBS using rTMS induces disinhibition of ipsilesional motor cortex that contributes to neural plasticity by unmasking latent networks [20]. Therefore, bilateral NIBS may more effectively facilitate neural plasticity and induce motor recovery after stroke.

In addition to motor recovery, bilateral NIBS can protect against the deterioration of bimanual movement caused by inhibitory NIBS over the unaffected hemisphere. Inhibitory NIBS might worsen the antiphase bimanual movement by reducing the interhemispheric inhibition that controls bimanual movement [42]. However, a combination of inhibitory NIBS over the unaffected hemisphere and excitatory NIBS over the affected hemisphere could prevent this deterioration by decreasing the reduction of interhemispheric inhibition (Figure 2(b)) [42]. It has been suggested that activation of inhibitory interneurons in the affected hemisphere by excitatory NIBS may limit the reduction of interhemispheric inhibition from the unaffected to the affected hemisphere in bilateral NIBS protocols [42]. Therefore, clinicians can consider bilateral NIBS for effective motor recovery and prevention of bimanual movement deterioration.

\section{Conclusion}

This paper focused on the underlying mechanisms of and future views on NIBS therapy in an effort to suggest approaches for appropriate cortical reorganization in stroke patients. Considering the interhemispheric competition model, improvement in motor deficits can be achieved by increasing the excitability of the affected hemisphere using excitatory NIBS or decreasing the excitability of the unaffected hemisphere using inhibitory NIBS. Artificially modulating the neural network by NIBS may induce a more suitable environment for experience-dependent plasticity and interfere with maladaptive neural activation, which weakens motor function and limits motor recovery. Based on the current data, the best NIBS technique is still unclear. However, clinicians should select the NIBS type by considering the peculiarities and recent findings for NIBS therapy. The response of inhibitory NIBS over the unaffected hemisphere may be uniform, but it may deteriorate bimanual movement by reducing interhemispheric inhibition. Excitatory NIBS over the unaffected hemisphere may improve motor function for muscles that are strongly innervated by ipsilateral motor projections. The bilateral NIBS method may facilitate motor recovery more than unilateral NIBS and prevent the bimanual movement deterioration caused by inhibitory NIBS. Moreover, clinicians must consider that clinical factors, including age, stroke duration, lesion site, 
and severity of motor function loss, may influence the effects of NIBS therapy. NIBS can be applied as an adjuvant therapy with emerging neurorehabilitation strategies to enhance neural plasticity. However, very few of all possible combinations of NIBS and neurorehabilitation techniques have been tested experimentally. In addition to studies on optimum NIBS parameters, studies on combining NIBS with developed neurorehabilitation techniques to promote motor recovery after stroke should be conducted in the future.

\section{Acknowledgment}

This work was supported by JSPS Grant-in-Aid for Scientific Research Number 23650314.

\section{References}

[1] P. L. Kolominsky-Rabas, M. Weber, O. Gefeller, B. Neundoerfer, and P. U. Heuschmann, "Epidemiology of ischemic stroke subtypes according to TOAST criteria: incidence, recurrence, and long-term survival in ischemic stroke subtypes: a population-based study," Stroke, vol. 32, no. 12, pp. 27352740, 2001.

[2] G. Kwakkel, B. J. Kollen, and R. C. Wagenaar, "Long term effects of intensity of upper and lower limb training after stroke: a randomised trial," Journal of Neurology Neurosurgery and Psychiatry, vol. 72, no. 4, pp. 473-479, 2002.

[3] G. J. Hankey, K. Jamrozik, R. J. Broadhurst, S. Forbes, and C. S. Anderson, "Long-term disability after first-ever stroke and related prognostic factors in the Perth Community Stroke Study, 1989-1990," Stroke, vol. 33, no. 4, pp. 1034-1040, 2002.

[4] B. B. Johansson, "Current trends in stroke rehabilitation. A review with focus on brain plasticity," Acta Neurologica Scandinavica, vol. 123, no. 3, pp. 147-159, 2011.

[5] P. Langhorne, J. Bernhardt, and G. Kwakkel, "Stroke rehabilitation,” The Lancet, vol. 377, no. 9778, pp. 1693-1702, 2011.

[6] A. P. Reiss, S. L. Wolf, E. A. Hammel, E. L. McLeod, and E. A. Williams, "Constraint-induced movement therapy (CIMT): current perspectives and future directions," Stroke Research and Treatment, vol. 2012, Article ID 159391, 8 pages, 2012.

[7] J. A. Hosp and A. R. Luft, "Cortical plasticity during motor learning and recovery after ischemic stroke," Neural Plasticity, vol. 2011, Article ID 871296, 9 pages, 2011.

[8] E. Dayan and L. G. Cohen, "Neuroplasticity subserving motor skill learning," Neuron, vol. 72, no. 3, pp. 443-454, 2011.

[9] M. A. Maldonado, R. P. Allred, E. L. Felthauser, and T. A. Jones, "Motor skill training, but not voluntary exercise, improves skilled reaching after unilateral ischemic lesions of the sensorimotor cortex in rats," Neurorehabilitation and Neural Repair, vol. 22, no. 3, pp. 250-261, 2008.

[10] H. Chen, J. Epstein, and E. Stern, "Neural plasticity after acquired brain injury: evidence from functional neuroimaging," PM \& R, vol. 2, no. 12, pp. S306-S312, 2010.

[11] N. Dancause and R. J. Nudo, "Shaping plasticity to enhance recovery after injury," Progress in Brain Research, vol. 192, pp. 273-295, 2011.

[12] J. P. Lefaucheur, "Methods of therapeutic cortical stimulation," Neurophysiologie Clinique, vol. 39, no. 1, pp. 1-14, 2009.
[13] Y. Z. Huang, M. J. Edwards, E. Rounis, K. P. Bhatia, and J. C. Rothwell, "Theta burst stimulation of the human motor cortex," Neuron, vol. 45, no. 2, pp. 201-206, 2005.

[14] S. Zaghi, M. Acar, B. Hultgren, P. S. Boggio, and F. Fregni, "Noninvasive brain stimulation with low-intensity electrical currents: putative mechanisms of action for direct and alternating current stimulation," Neuroscientist, vol. 16, no. 3, pp. 285-307, 2010.

[15] M. A. Nitsche and W. Paulus, "Excitability changes induced in the human motor cortex by weak transcranial direct current stimulation," Journal of Physiology, vol. 527, no. 3, pp. 633-639, 2000.

[16] N. Takeuchi and S. -I. Izumi, "Maladaptive plasticity for motor recovery after stroke: mechanisms and approaches," Neural Plasticity, vol. 2012, Article ID 359728, 9 pages, 2012.

[17] N. Murase, J. Duque, R. Mazzocchio, and L. G. Cohen, "Influence of interhemispheric interactions on motor function in chronic stroke," Annals of Neurology, vol. 55, no. 3, pp. 400-409, 2004.

[18] N. Takeuchi, T. Chuma, Y. Matsuo, I. Watanabe, and K. Ikoma, "Repetitive transcranial magnetic stimulation of contralesional primary motor cortex improves hand function after stroke," Stroke, vol. 36, no. 12, pp. 2681-2686, 2005.

[19] D. A. Nowak, C. Grefkes, M. Ameli, and G. R. Fink, "Interhemispheric competition after stroke: brain stimulation to enhance recovery of function of the affected hand," Neurorehabilitation and Neural Repair, vol. 23, no. 7, pp. 641656, 2009.

[20] N. Takeuchi, T. Tada, M. Toshima, Y. Matsuo, and K. Ikoma, "Repetitive transcranial magnetic stimulation over bilateral hemispheres enhances motor function and training effect of paretic hand in patients after stroke," Journal of Rehabilitation Medicine, vol. 41, no. 13, pp. 1049-1054, 2009.

[21] C. Poreisz, K. Boros, A. Antal, and W. Paulus, "Safety aspects of transcranial direct current stimulation concerning healthy subjects and patients," Brain Research Bulletin, vol. 72, no. 46, pp. 208-214, 2007.

[22] S. Rossi, M. Hallett, P. M. Rossini, and A. Pascual-Leone, "Safety, ethical considerations, and application guidelines for the use of transcranial magnetic stimulation in clinical practice and research," Clinical Neurophysiology, vol. 120, no. 12, pp. 2008-2039, 2009.

[23] F. Hummel, P. Celnik, P. Giraux et al., "Effects of non-invasive cortical stimulation on skilled motor function in chronic stroke," Brain, vol. 128, no. 3, pp. 490-499, 2005.

[24] Y. H. Kim, S. H. You, M. H. Ko et al., "Repetitive transcranial magnetic stimulation-induced corticomotor excitability and associated motor skill acquisition in chronic stroke," Stroke, vol. 37, no. 6, pp. 1471-1476, 2006.

[25] N. Takeuchi, T. Tada, M. Toshima, T. Chuma, Y. Matsuo, and K. Ikoma, "Inhibition of the unaffected motor cortex by $1 \mathrm{HZ}$ repetitive transcranial magnetic stimulation enhances motor performance and training effect of the paretic hand in patients with chronic stroke," Journal of Rehabilitation Medicine, vol. 40, no. 4, pp. 298-303, 2008.

[26] V. Di Lazzaro, P. Profice, F. Pilato et al., "Motor cortex plasticity predicts recovery in acute stroke," Cerebral Cortex, vol. 20, no. 7, pp. 1523-1528, 2010.

[27] A. Pascual-Leone, F. Tarazona, J. Keenan, J. M. Tormos, R. Hamilton, and M. D. Catala, "Transcranial magnetic stimulation and neuroplasticity," Neuropsychologia, vol. 37, no. 2, pp. 207-217, 1998. 
[28] W. Muellbacher, U. Zlemann, J. Wissel et al., "Early consolidation in human primary motor cortex," Nature, vol. 415, no. 6872 , pp. 640-644, 2002.

[29] F. Conchou, I. Loubinoux, E. Castel-Lacanal et al., "Neural substrates of low-frequency repetitive transcranial magnetic stimulation during movement in healthy subjects and acute stroke patients. A PET study," Human Brain Mapping, vol. 30, no. 8, pp. 2542-2557, 2009.

[30] M. Ameli, C. Grefkes, F. Kemper et al., "Differential effects of high-frequency repetitive transcranial magnetic stimulation over ipsilesional primary motor cortex in cortical and subcortical middle cerebral artery stroke," Annals of Neurology, vol. 66, no. 3, pp. 298-309, 2009.

[31] S. T. Carmichael, "Cellular and molecular mechanisms of neural repair after stroke: making waves," Annals of Neurology, vol. 59, no. 5, pp. 735-742, 2006.

[32] M. Di Filippo, A. Tozzi, C. Costa et al., "Plasticity and repair in the post-ischemic brain," Neuropharmacology, vol. 55, no. 3, pp. 353-362, 2008.

[33] E. M. Khedr, M. A. Ahmed, N. Fathy, and J. C. Rothwell, "Therapeutic trial of repetitive transcranial magnetic stimulation after acute ischemic stroke," Neurology, vol. 65, no. 3, pp. 466-468, 2005.

[34] F. Fregni, P. S. Boggio, A. C. Valle et al., "A sham-controlled trial of a 5-day course of repetitive transcranial magnetic stimulation of the unaffected hemisphere in stroke patients," Stroke, vol. 37, no. 8, pp. 2115-2122, 2006.

[35] D. A. Nowak, C. Grefkes, M. Dafotakis et al., "Effects of low-frequency repetitive transcranial magnetic stimulation of the contralesional primary motor cortex on movement kinematics and neural activity in subcortical stroke," Archives of Neurology, vol. 65, no. 6, pp. 741-747, 2008.

[36] C. Grefkes, D. A. Nowak, L. E. Wang, M. Dafotakis, S. B. Eickhoff, and G. R. Fink, "Modulating cortical connectivity in stroke patients by rTMS assessed with fMRI and dynamic causal modeling," NeuroImage, vol. 50, no. 1, pp. 233-242, 2010.

[37] N. Takeuchi and K. Ikoma, "1 Hz rTMS over unaffected hemisphere in stroke patients alters bilateral movements and coupling between motor areas," Clinical Neurophysiology, vol. 121, supplement 1, p. s316, 2010.

[38] N. S. Ward and R. S. J. Frackowiak, "The functional anatomy of cerebral reorganisation after focal brain injury," Journal of Physiology Paris, vol. 99, no. 4-6, pp. 425-436, 2006.

[39] P. Talelli, R. J. Greenwood, and J. C. Rothwell, "Arm function after stroke: neurophysiological correlates and recovery mechanisms assessed by transcranial magnetic stimulation," Clinical Neurophysiology, vol. 117, no. 8, pp. 1641-1659, 2006.

[40] W. Y. Hsu, C. H. Cheng, K. K. Liao, I. H. Lee, and Y. Y. Lin, "Effects of repetitive transcranial magnetic stimulation on motor functions in patients with stroke: a meta-analysis," Stroke, vol. 43, no. 7, pp. 1849-1857, 2012.

[41] S. Kumar, C. W. Wagner, C. Frayne et al., "Noninvasive brain stimulation may improve stroke-related dysphagia: a pilot study," Stroke, vol. 42, no. 4, pp. 1035-1040, 2011.

[42] N. Takeuchi, T. Tada, Y. Matsuo, and K. Ikoma, "Lowfrequency repetitive TMS plus anodal transcranial DCS prevents transient decline in bimanual movement induced by contralesional inhibitory rTMS after stroke," Neurorehabilitation and Neural Repair, vol. 26, no. 8, pp. 988-998, 2012.
[43] A. Turton, S. Wroe, N. Trepte, C. Fraser, and R. N. Lemon, "Contralateral and ipsilateral EMG responses to transcranial magnetic stimulation during recovery of arm and hand function after stroke," Electroencephalography and Clinical Neurophysiology, vol. 101, no. 4, pp. 316-328, 1996.

[44] J. Netz, T. Lammers, and V. Hömberg, "Reorganization of motor output in the non-affected hemisphere after stroke," Brain, vol. 120, no. 9, pp. 1579-1586, 1997.

[45] K. J. Werhahn, A. B. Conforto, N. Kadom, M. Hallett, and L. G. Cohen, "Contribution of the ipsilateral motor cortex to recovery after chronic stroke," Annals of Neurology, vol. 54, no. 4, pp. 464-472, 2003.

[46] E. Palmer and P. Ashby, "Corticospinal projections to upper limb motoneurones in humans," Journal of Physiology, vol. 448, pp. 397-412, 1992.

[47] P. Bawa, J. D. Hamm, P. Dhillon, and P. A. Gross, "Bilateral responses of upper limb muscles to transcranial magnetic stimulation in human subjects," Experimental Brain Research, vol. 158, no. 3, pp. 385-390, 2004.

[48] W. Muellbacher, C. Artner, and B. Mamoli, "The role of the intact hemisphere in recovery of midline muscles after recent monohemispheric stroke," Journal of Neurology, vol. 246, no. 4, pp. 250-256, 1999.

[49] J. A. Eyre, J. P. Taylor, F. Villagra, M. Smith, and S. Miller, "Evidence of activity-dependent withdrawal of corticospinal projections during human development," Neurology, vol. 57, no. 9, pp. 1543-1554, 2001.

[50] C. Gerloff, C. Braun, M. Staudt, Y. L. Hegner, J. Dichgans, and I. Krägeloh-Mann, "Coherent corticomuscular oscillations originate from primary motor cortex: evidence from patients with early brain lesions," Human Brain Mapping, vol. 27, no. 10, pp. 789-798, 2006.

[51] C. Gerloff and F. G. Andres, "Bimanual coordination and interhemispheric interaction," Acta psychologica, vol. 110, no. 2-3, pp. 161-186, 2002.

[52] A. Stančák, E. R. Cohen, R. D. Seidler, T. Q. Duong, and S. G. Kim, "The size of corpus callosum correlates with functional activation of medial motor cortical areas in bimanual and unimanual movements," Cerebral Cortex, vol. 13, no. 5, pp. 475-485, 2003.

[53] T. Wagner, F. Fregni, U. Eden et al., "Transcranial magnetic stimulation and stroke: a computer-based human model study," NeuroImage, vol. 30, no. 3, pp. 857-870, 2006.

[54] T. Wagner, A. Valero-Cabre, and A. Pascual-Leone, "Noninvasive human brain stimulation," Annual Review of Biomedical Engineering, vol. 9, pp. 527-565, 2007.

[55] S. -I. Izumi, Y. Oouchida, T. Okita et al., "Development and utility of an integrating circuit to measure a pulsed magnetic field in comparison with its theoretical magnetic field structure," Japanese Journal of Comprehensive Rehabilitation. In press.

[56] T. Touge, W. Gerschlager, P. Brown, and J. C. Rothwell, "Are the after-effects of low-frequency rTMS on motor cortex excitability due to changes in the efficacy of cortical synapses?" Clinical Neurophysiology, vol. 112, no. 11, pp. 2138-2145, 2001.

[57] P. B. Fitzgerald, T. L. Brown, Z. J. Daskalakis, R. Chen, and J. Kulkarni, "Intensity-dependent effects of $1 \mathrm{~Hz}$ rTMS on human corticospinal excitability," Clinical Neurophysiology, vol. 113, no. 7, pp. 1136-1141, 2002.

[58] A. Peinemann, B. Reimer, C. Löer et al., "Long-lasting increase in corticospinal excitability after 1800 pulses of 
subthreshold $5 \mathrm{~Hz}$ repetitive TMS to the primary motor cortex," Clinical Neurophysiology, vol. 115, no. 7, pp. 15191526, 2004.

[59] M. B. Iyer, U. Mattu, J. Grafman, M. Lomarev, S. Sato, and E. M. Wassermann, "Safety and cognitive effect of frontal DC brain polarization in healthy individuals," Neurology, vol. 64, no. 5, pp. 872-875, 2005.

[60] H. M. Schambra, L. Sawaki, and L. G. Cohen, "Modulation of excitability of human motor cortex (M1) by $1 \mathrm{~Hz}$ transcranial magnetic stimulation of the contralateral M1," Clinical Neurophysiology, vol. 114, no. 1, pp. 130-133, 2003.

[61] K. J. Werhahn, A. B. Conforto, N. Kadom, M. Hallett, and L. G. Cohen, "Contribution of the ipsilateral motor cortex to recovery after chronic stroke," Annals of Neurology, vol. 54, no. 4, pp. 464-472, 2003.

[62] M. Kobayashi, S. Hutchinson, H. Théoret, G. Schlaug, and A. Pascual-Leone, "Repetitive TMS of the motor cortex improves ipsilateral sequential simple finger movements," Neurology, vol. 62, no. 1, pp. 91-98, 2004.

[63] N. Yozbatiran, M. Alonso-Alonso, J. See et al., "Safety and behavioral effects of high-frequency repetitive transcranial magnetic stimulation in stroke," Stroke, vol. 40, no. 1, pp. 309-312, 2009.

[64] A. Kirton, R. Chen, S. Friefeld, C. Gunraj, A. M. Pontigon, and G. deVeber, "Contralesional repetitive transcranial magnetic stimulation for chronic hemiparesis in subcortical paediatric stroke: a randomised trial," The Lancet Neurology, vol. 7, no. 6, pp. 507-513, 2008.

[65] G. H. Moll, H. Heinrich, S. Wischer, F. Tergau, W. Paulus, and A. Rothenberger, "Motor system excitability in healthy children: developmental aspects from transcranial magnetic stimulation," Electroencephalography and Clinical Neurophysiology, Supplement, vol. 51, pp. 243-249, 1999.

[66] M. C. Ridding and U. Ziemann, "Determinants of the induction of cortical plasticity by non-invasive brain stimulation in healthy subjects," Journal of Physiology, vol. 588, no. 13, pp. 2291-2304, 2010.

[67] L. Sawaki, Z. Yaseen, L. Kopylev, and L. G. Cohen, "Agedependent changes in the ability to encode a novel elementary motor memory," Annals of Neurology, vol. 53, no. 4, pp. 521-524, 2003.

[68] J. Liepert, S. Zittel, and C. Weiller, "Improvement of dexterity by single session low-frequency repetitive transcranial magnetic stimulation over the contralesional motor cortex in acute stroke: a double-blind placebo-controlled crossover trial," Restorative Neurology and Neuroscience, vol. 25, no. 5-6, pp. 461-465, 2007.

[69] E. M. Khedr, M. R. Abdel-Fadeil, A. Farghali, and M. Qaid, "Role of 1 and $3 \mathrm{~Hz}$ repetitive transcranial magnetic stimulation on motor function recovery after acute ischaemic stroke," European Journal of Neurology, vol. 16, no. 12, pp. 1323-1330, 2009.

[70] E. M. Khedr, A. E. Etraby, M. Hemeda, A. M. Nasef, and A. A. E. Razek, "Long-term effect of repetitive transcranial magnetic stimulation on motor function recovery after acute ischemic stroke," Acta Neurologica Scandinavica, vol. 121, no. 1, pp. 30-37, 2010.

[71] J. Seniów, M. Bilik, M. Lesniak, K. Waldowski, S. Iwanski, and A. Czlonkowska, "Transcranial magnetic stimulation combined with physiotherapy in rehabilitation of poststroke hemiparesis: a randomized, double-blind, placebocontrolled study," Neurorehabilitation Neural and Repair. In press.
[72] A. Pascual-Leone, A. Amedi, F. Fregni, and L. B. Merabet, "The plastic human brain cortex," Annual Review of Neuroscience, vol. 28, pp. 377-401, 2005.

[73] B. Picconi, A. Tortiglione, I. Barone et al., "NR2B subunit exerts a critical role in postischemic synaptic plasticity," Stroke, vol. 37, no. 7, pp. 1895-1901, 2006.

[74] F. Fregni, P. S. Boggio, C. G. Mansur et al., "Transcranial direct current stimulation of the unaffected hemisphere in stroke patients," NeuroReport, vol. 16, no. 14, pp. 1551-1555, 2005.

[75] P. Talelli, R. J. Greenwood, and J. C. Rothwell, "Exploring Theta Burst Stimulation as an intervention to improve motor recovery in chronic stroke," Clinical Neurophysiology, vol. 118, no. 2, pp. 333-342, 2007.

[76] P. S. Boggio, M. Alonso-Alonso, C. G. Mansur et al., "Hand function improvement with low-frequency repetitive transcranial magnetic stimulation of the unaffected hemisphere in a severe case of stroke," American Journal of Physical Medicine and Rehabilitation, vol. 85, no. 11, pp. 927-930, 2006.

[77] J. Málly and E. Dinya, "Recovery of motor disability and spasticity in post-stroke after repetitive transcranial magnetic stimulation (rTMS)," Brain Research Bulletin, vol. 76, no. 4, pp. 388-395, 2008.

[78] S. I. Izumi, T. Kondo, and K. Shindo, "Transcranial magnetic stimulation synchronized with maximal movement effort of the hemiplegic hand after stroke: a double-blinded controlled pilot study," Journal of Rehabilitation Medicine, vol. 40, no. 1, pp. 49-54, 2008.

[79] S. Tanaka, K. Takeda, Y. Otaka et al., "Single session of transcranial direct current stimulation transiently increases knee extensor force in patients with hemiparetic stroke," Neurorehabilitation and Neural Repair, vol. 25, no. 6, pp. 565$569,2011$.

[80] S. Madhavan, K. A. Weber, and J. W. Stinear, "Non-invasive brain stimulation enhances fine motor control of the hemiparetic ankle: implications for rehabilitation," Experimental Brain Research, vol. 209, no. 1, pp. 9-17, 2011.

[81] G. Jayaram and J. W. Stinear, "The effects of transcranial stimulation on paretic lower limb motor excitability during walking," Journal of Clinical Neurophysiology, vol. 26, no. 4, pp. 272-279, 2009.

[82] M. Hallett, "Surround inhibition," Supplements to Clinical Neurophysiology, vol. 56, pp. 153-159, 2003.

[83] D. T. Jeffery, J. A. Norton, F. D. Roy, and M. A. Gorassini, "Effects of transcranial direct current stimulation on the excitability of the leg motor cortex," Experimental Brain Research, vol. 182, no. 2, pp. 281-287, 2007.

[84] J. R. Carey, C. D. Evans, D. C. Anderson et al., "Safety of 6-Hz primed low-frequency rTMS in stroke," Neurorehabilitation and Neural Repair, vol. 22, no. 2, pp. 185-192, 2008.

[85] R. Lindenberg, V. Renga, L. L. Zhu, D. Nair, and G. Schlaug, "Bihemispheric brain stimulation facilitates motor recovery in chronic stroke patients," Neurology, vol. 75, no. 24, pp. 2176-2184, 2010.

[86] H. Mahmoudi, A. B. Haghighi, P. Petramfar, S. Jahanshahi, Z. Salehi, and F. Fregni, "Transcranial direct current stimulation: electrode montage in stroke," Disability and Rehabilitation, vol. 33, no. 15-16, pp. 1383-1388, 2011.

[87] S. Hesse, C. Werner, E. M. Schonhardt, A. Bardeleben, W. Jenrich, and S. G. B. Kirker, "Combined transcranial direct current stimulation and robot-assisted arm training in subacute stroke patients: a pilot study," Restorative Neurology and Neuroscience, vol. 25, no. 1, pp. 9-15, 2007. 
[88] D. J. Edwards, H. I. Krebs, A. Rykman et al., "Raised corticomotor excitability of M1 forearm area following anodal tDCS is sustained during robotic wrist therapy in chronic stroke," Restorative Neurology and Neuroscience, vol. 27, no. 3, pp. 199-207, 2009.

[89] P. Celnik, N. J. Paik, Y. Vandermeeren, M. Dimyan, and L. G. Cohen, "Effects of combined peripheral nerve stimulation and brain polarization on performance of a motor sequence task after chronic stroke," Stroke, vol. 40, no. 5, pp. 17641771, 2009.

[90] N. Bolognini, G. Vallar, C. Casati et al., "Neurophysiological and behavioral effects of tDCS combined with constraintinduced movement therapy in poststroke patients," Neurorehabilitation Neural and Repair, vol. 25, no. 9, pp. 819-829, 2011.

[91] M. P. Malcolm, W. J. Triggs, K. E. Light et al., "Repetitive transcranial magnetic stimulation as an adjunct to constraint-induced therapy: an exploratory randomized controlled trial," American Journal of Physical Medicine and Rehabilitation, vol. 86, no. 9, pp. 707-715, 2007.

[92] S. Theilig, J. Podubecka, K. Bösl, R. Wiederer, and D. A. Nowak, "Functional neuromuscular stimulation to improve severe hand dysfunction after stroke: does inhibitory rTMS enhance therapeutic efficiency?" Experimental Neurology, vol. 230, no. 1, pp. 149-155, 2011.

[93] U. Ziemann, F. Meintzschel, A. Korchounov, and T. V. Ilić, "Pharmacological modulation of plasticity in the human motor cortex," Neurorehabilitation and Neural Repair, vol. 20, no. 2, pp. 243-251, 2006.

[94] M. A. Nitsche, W. Jaussi, D. Liebetanz, N. Lang, F. Tergau, and W. Paulus, "Consolidation of human motor cortical neuroplasticity by D-cycloserine," Neuropsychopharmacology, vol. 29, no. 8, pp. 1573-1578, 2004.

[95] M. F. Kuo, W. Paulus, and M. A. Nitsche, "Boosting focallyinduced brain plasticity by dopamine," Cerebral Cortex, vol. 18, no. 3, pp. 648-651, 2008.

[96] M. A. Nitsche, C. Lampe, A. Antal et al., "Dopaminergic modulation of long-lasting direct current-induced cortical excitability changes in the human motor cortex," European Journal of Neuroscience, vol. 23, no. 6, pp. 1651-1657, 2006.

[97] N. Lang, S. Speck, J. Harms, H. Rothkegel, W. Paulus, and M. Sommer, "Dopaminergic potentiation of rTMS-induced motor cortex inhibition," Biological Psychiatry, vol. 63, no. 2, pp. 231-233, 2008.

[98] O. B. C. Swayne, J. T. H. Teo, R. J. Greenwood, and J. C. Rothwell, "The facilitatory effects of intermittent theta burst stimulation on corticospinal excitability are enhanced by nicotine," Clinical Neurophysiology, vol. 120, no. 8, pp. 1610$1615,2009$.

[99] M. A. Nitsche, J. Grundey, D. Liebetanz, N. Lang, F. Tergau, and W. Paulus, "Catecholaminergic consolidation of motor cortical neuroplasticity in humans," Cerebral Cortex, vol. 14, no. 11, pp. 1240-1245, 2004.

[100] M. A. Nitsche, M. F. Kuo, R. Karrasch, B. Wächter, D. Liebetanz, and W. Paulus, "Serotonin affects transcranial direct current-induced neuroplasticity in humans," Biological Psychiatry, vol. 66, no. 5, pp. 503-508, 2009. 


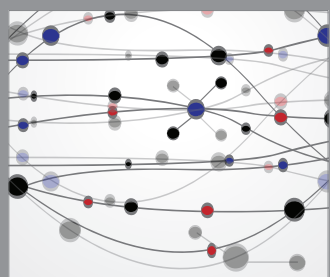

The Scientific World Journal
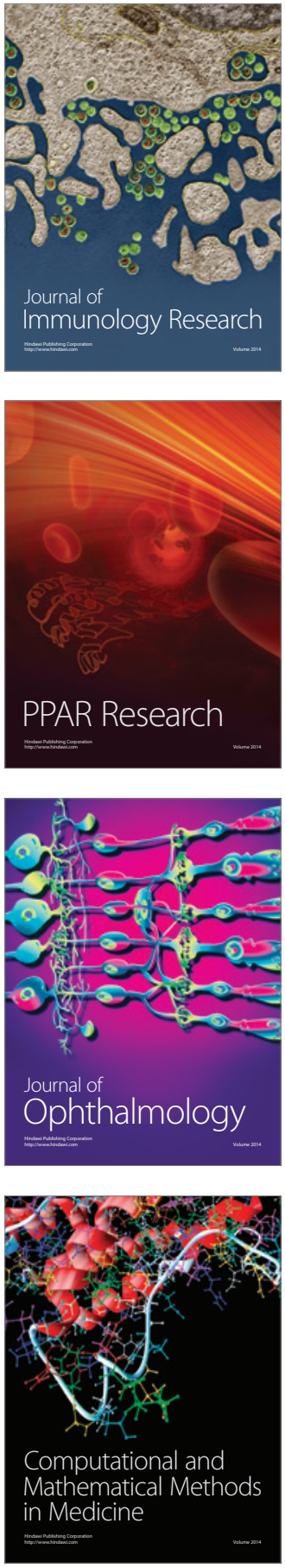

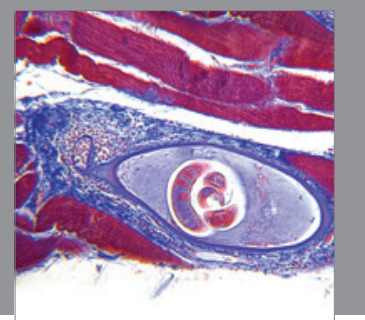

Gastroenterology

Research and Practice
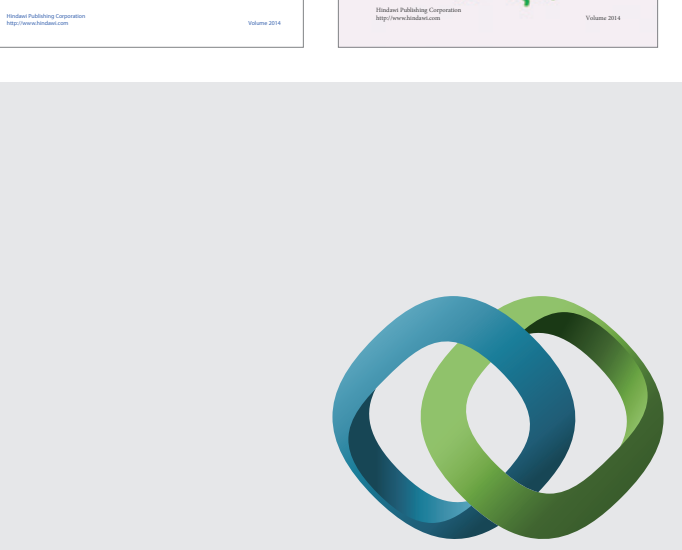

\section{Hindawi}

Submit your manuscripts at

http://www.hindawi.com
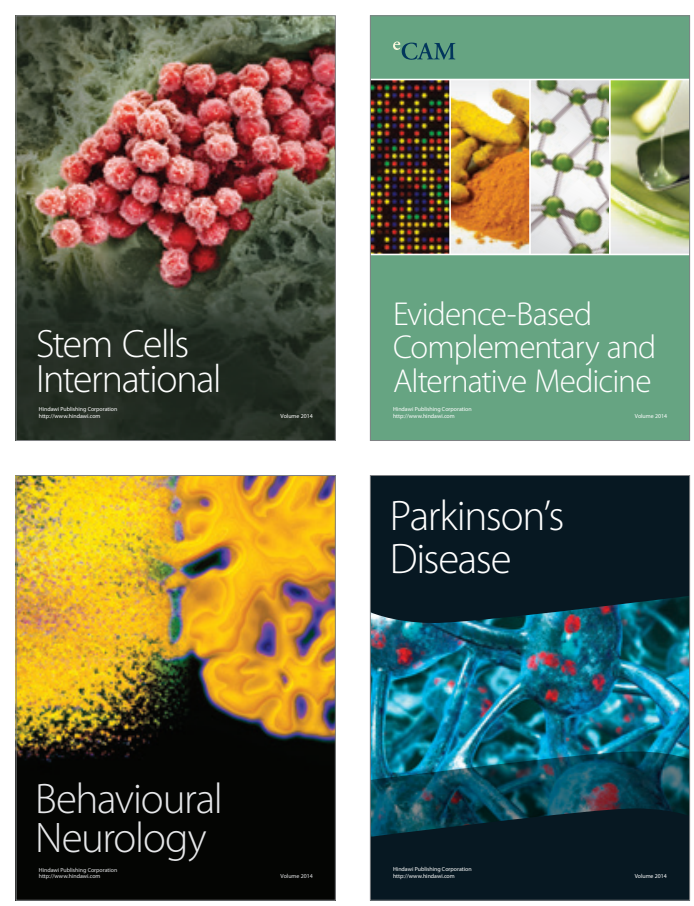

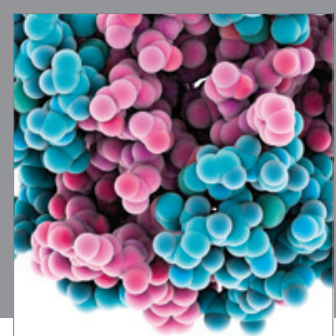

Journal of
Diabetes Research

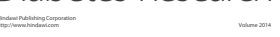

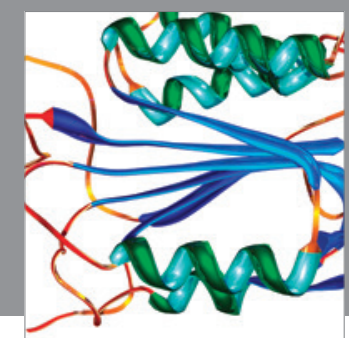

Disease Markers
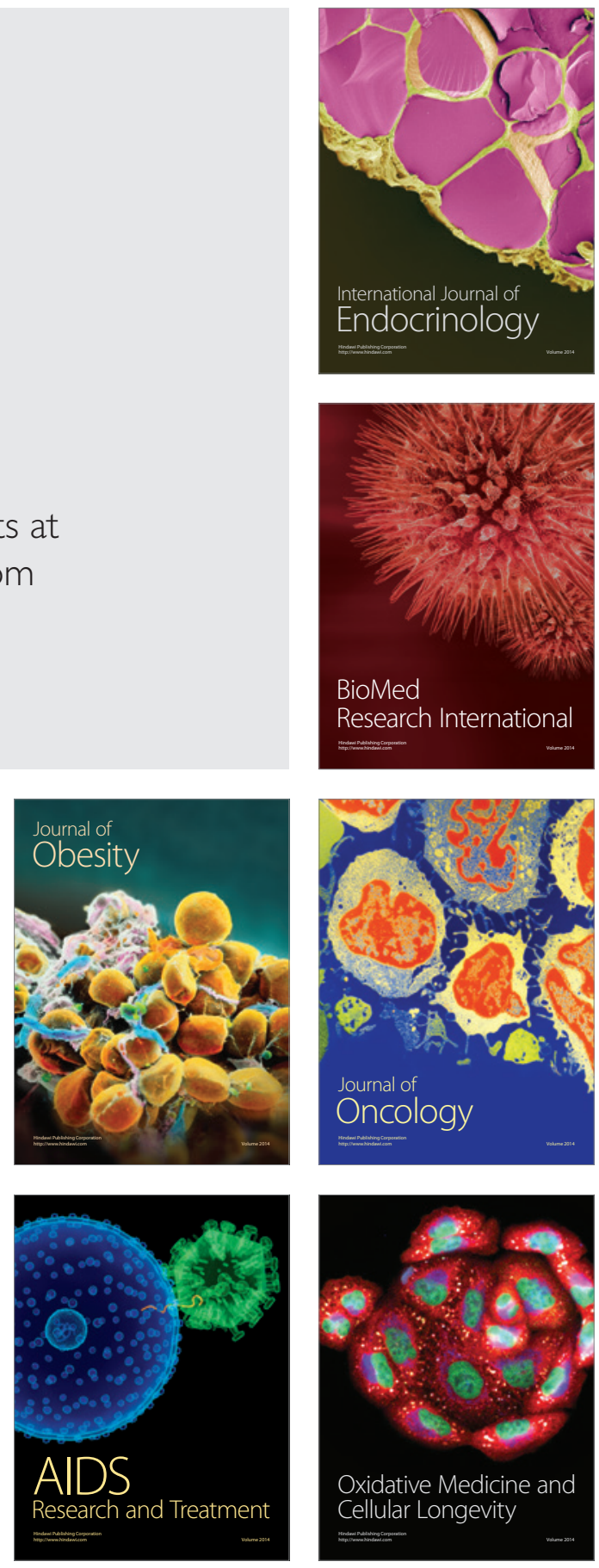